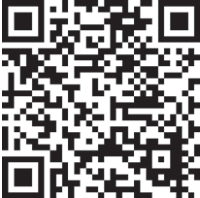

* Profesor Médico del Centro de Investigación Educativa y Formación Docente del Centro Médico Nacional «Siglo XX|» del Instituto Mexicano del Seguro Social.

₹ Médico Residente de la Especialidad de Medicina Familiar en la UMF Núm. 49, Celaya, Guanajuato.

Correspondencia: FHP, mesias.francisco@gmail. com

Conflicto de intereses: Los autores declaran que no tienen. Citar como: Hernández Pérez F, Bustillos Hernández DZ. Percepción del ambiente educativo y el maltrato de médicos residentes de medicina familiar. Rev CONAMED 2020; 25(1): 10-15. doi: $10.35366 / 92890$ Financiamiento: Este trabajo no contó con financiamiento.

Recibido: 09/12/2019 Aceptado: 04/03/2020.

\section{Percepción del ambiente educativo y el maltrato de médicos residentes de medicina familiar}

\author{
Perception of the educational environment and abuse of resident of family \\ Francisco Hernández Pérez,* Deyanira Zareth Bustillos Hernández
}

\section{RESUMEN}

Introducción: Los ambientes educativos son de vital influencia en los resultados a corto y largo plazo del aprendizaje; no obstante, éstos, con frecuencia, no están exentos de agresiones u hostilidades. Objetivo: Identificar la percepción del ambiente educativo y frecuencia del maltrato en médicos residentes de medicina familiar. Material y métodos: Estudio transversal y analítico realizado en un grupo natural de 66 médicos residentes. Para ello, se utilizó la encuesta PHEEM (Postgraduate Hospital Educational Environment Measure) con el objetivo de evaluar el ambiente educativo y la escala de maltrato, la cual ha sido validada por Mejía y colaboradores. Resultados: Se estudiaron 52 residentes de primer, segundo y tercer año del Hospital General Núm. 47 de Celaya, Guanajuato y, de acuerdo con la evaluación, la percepción del ambiente educativo global fue de 85.3, es decir, un ambiente más positivo que negativo. Sin embargo, al considerar por separado los diferentes grados, sólo los residentes de tercer año consideraron su ambiente más favorable que negativo (102.50). Por su parte, los de primer y segundo año, lo percibieron como un ambiente con muchos problemas $(80.49$ y 77.21 , respectivamente, con una diferencia significativa entre los tres grupos [ $p=0001])$. La frecuencia de maltrato sólo fue positiva en 15\%. Conclusiones: En el presente estudio, los médicos residentes perciben a su ambiente educativo, en general, como bueno (más positivo que negativo), pero refieren también una necesidad de mejor capacitación. En cuanto al maltrato, la frecuencia es baja con apenas 15\% global, la cual se incrementa en el tercer año de residencia.

Palabras clave: Ambiente educativo, maltrato, residentes.

\begin{abstract}
Introduction: Educational environments have a vital influence on short and long term educational outcomes; often these are not exempt from aggressions or hostilities. Objective: To identify the perception of the educational environment and frequency of abuse in family medicine residents. Material and methods: Analytical cross-sectional study, in a natural group of 66 resident physicians, the PHEEM (Postgraduate Hospital Educational Environment Measure) survey will be used to assess the educational environment and the scale of abuse of Mejía et al. Results: 52 first, second and third year residents of the General Hospital No. 47 of Celaya, Guanajuato were studied, the perception of global educational environment was 85.3 (educational environment more positive than negative), however, when considering the different grades separately, only third-year residents consider their educational environment more favorable than negative (102.50), while first and second-year residents perceive it as an educational environment with many problems (80.49 and 77.21$)$ respectively, finding a significant difference between the three groups $(p=0001)$. The frequency of abuse was only positive in $15 \%$. Conclusions: In the present study, resident physicians perceive their general educational environment as good (more positive than negative) with a need for better training. As for abuse, the frequency is low, barely $15 \%$ overall, which increases in the third year of residence.
\end{abstract}

Keywords: Educational environment, abuse, residents. 


\section{INTRODUCCIÓN}

El «ambiente educativo» es producto del ambiente físico, de las relaciones interpersonales, del estilo de comunicación dominante, de las presiones y factores estresantes, del sistema de reconocimientos y de las sanciones en los espacios educativos, cuya interacción afecta de una u otra forma el rendimiento y la satisfacción de los estudiantes.-3

Todos los procesos educativos tienen condiciones únicas e irrepetibles, lo que se conoce como «cultura del lugar» o habitus, el cual no es explícito, sino que es parte de un «currículo oculto» no planificado. En este sentido, la evaluación de estos espacios no se vuelve sencilla ni fácil, por lo que se suele recurrir a la percepción que los estudiantes tienen sobre el contexto en el que se desarrolla la actividad educativa más allá de la visión oficial, donde pareciera que sólo se privilegian sus potenciales y capacidades; pese a ello, la escuela suele ser el lugar en el que más se reproducen las relaciones de poder y contradicciones que de ésta emanan, ya que todo sistema educativo tiene que reproducirlas y legitimarlas con sus propios medios. ${ }^{1-8}$

En México, existe la Norma Oficial Mexicana para la Regulación Normativa de las Residencias Médicas, ${ }^{9}$ la cual establece las jornadas laborales, la periodicidad de las guardias, así como los derechos con los que cuentan los médicos residentes y sus obligaciones; sin embargo, estas normas no siempre son cumplidas. Como se sabe, los ambientes educativos tienen una influencia vital en los resultados educativos a corto y largo plazo. Según diversos estudios, la prevalencia de maltrato académico y físico oscila entre 60 y 95\%, aunque esta conducta se ha «normalizado», y esta «normalización» ha llevado a crear el concepto de que la violencia es inherente a la formación del médico. Asimismo, se ha observado que esta percepción de maltrato físico y académico se relaciona de manera inversa con la percepción de aprendizaje. Por este razonamiento es que se pone en duda el hecho de que si no existe la violencia no se aprende, ya que se ha visto que en el ambiente educativo sucede todo lo contrario, y que gran porcentaje de estudiantes de medicina o médicos residentes han renunciado como consecuencia de esta experiencia traumática, la cual Ileva a la pérdida de la motivación, sentimientos de inadecuación y fracaso, cansancio emocional, despersonalización y baja relación en el trabajo. ${ }^{10-17}$

El proceso de un buen aprendizaje debe estar basado en un equilibrio entre el adecuado ambiente académico y el social; es evidente que los entornos laborales tradicionales poco reflexivos no sólo no contribuyen al desarrollo de aptitudes en los residentes, sino que pueden ser fuentes de conflictos entre sus integrantes. Dicho lo anterior, nos lleva a redefinir sus características para que se contribuya a generar un ambiente propicio para la reflexión y bienestar del médico residente, y que éste redunde con un mayor grado en la calidad de los servicios prestados, ya que en ambientes adversos se genera una serie de conflictos en las formas de convivencia entre los que participan: profesores, alumnos y autoridades académicas; todo ello finalmente se verá reflejado en la calidad de atención. ${ }^{18-23}$

Existe un gran número de investigaciones que dan cuenta de que los médicos tienen una percepción muy mala de su entorno, particularmente en lo que se refiere a los abusos y violación de sus derechos. Estas mismas investigaciones también señalan que los estudiantes perciben al docente como una problemática, puesto que, en muchas ocasiones, éstos tienden a ser autoritarios y crean un ambiente poco propicio para la reflexión, el debate y el ejercicio de la crítica. 1,4,10,11,18,24,25

\section{MATERIAL Y MÉTODOS}

Se realizó un estudio observacional de tipo transversal con una muestra de médicos residentes del Curso de especialización de medicina familiar de la UMF 49 de Celaya, Guanajuato, durante el año lectivo de 2019. Se invitó al total de médicos residentes (66 residentes); de éstos, 52 aceptaron la invitación y fueron quienes cumplieron los criterios de inclusión, a saber: que aceptaran participar y que no estuvieran bajo tratamiento psicológico o que no hubieran entablado algún tipo de demanda contra sus profesores o el instituto. Se excluyó a los voluntarios con instrumentos mal llenados.

Se consideró el ambiente educativo, es decir, la percepción que tenía el alumno del entorno donde llevaba a cabo sus labores académicas, y se tomó como maltrato físico al empleo de la fuerza 
física contra una persona o grupo, produciendo daños físicos, sexuales o psicológicos, incluyendo en ellas golpes, empujones, pellizcos, violencia mediante el lanzamiento de un objeto a otra persona, asignación de trabajos excesivos o exposición a procedimientos sin la protección adecuada; el maltrato académico se consideró cuando se refirieron aquellas conductas inadecuadas por parte del docente hacia el alumno en el contexto del proceso enseñanza-aprendizaje que pudieran afectar su desempeño y su evaluación.

Los instrumentos de aplicación empleados fueron, en primer lugar, la escala Postgraduate Hospital Educational Environment Measure (PHEEM, por sus siglas en inglés) con una confiabilidad de 0.934 para evaluar la percepción del ambiente educativo. La escala pondera el ambiente en cuatro categorías: de 0-40 como ambiente educativo muy pobre; de 41-80 como ambiente educacional con muchos problemas; de 81-120 como ambiente educacional más positivo que negativo, pero con espacio para mejorar, y de 121-160 como ambiente educacional excelente.

Asimismo, la escala tiene cuatro subdominios: 1) percepción del rol de autonomía: de 0-14 como muy pobre; de 15-28 como una visión negativa del rol de cada uno; de 29-42 como una percepción más positiva del rol de cada uno, y de 43-56 como una percepción excelente del trabajo de cada uno (ítems 1, 4, 5, 8, 9, 11, 14, 17, 18, 29, 30, 32, 34 y 40). 2) Percepción de la enseñanza: de 0-15 como calidad muy pobre; de 16-30 como una necesidad de algo de reentrenamiento; de 31-45 como encaminado en la dirección correcta, y de 46-60 como enseñanza con maestros modelos (ítems 2, 3, 6, 10, 12 , 15, 21, 22, 23, 27, 28, 31, 33, 37 y 39). 3) Percepción del soporte social: de 0-11 como inexistente; de 12-22 como un lugar no placentero; de 23-33 como ambiente con más pros que contras, y de 34-44 como ambiente bueno y apoyador (ítems 7, 13, 16, 19, 20, $24,25,26,35,36$ y 38 ).

Para evaluar la frecuencia de maltrato, se utilizó, en segundo lugar, la escala de maltrato a residentes, la cual ha sido validada por Mejía y colaboradores, e incluye 13 situaciones de maltrato.

Para el análisis estadístico se utilizaron reporte de frecuencias, medias y desviación estándar, y para la estadística inferencial se utilizó ANOVA de un factor para el análisis de tres medias de distribución normal. Se consideró una $\mathrm{p}<0.05$ como estadísticamente significativa.

El presente trabajo se apegó a los principios éticos en investigación, el cual fue aprobado por el comité de ética local y contó con consentimiento informado de los participantes.

\section{RESULTADOS}

Se estudiaron 52 residentes de primer, segundo y tercer año. La edad promedio registrada fue $30.8 \pm$ 3.40 años, y el sexo registrado de los participantes fue 53.8\% de mujeres (Tabla 7).

Ambiente educativo. En cuanto al ambiente, los alumnos tuvieron un puntaje global de 85.38, lo que significa que consideran a su ambiente educativo más positivo que negativo. Asimismo, en el subdominio de autonomía, los alumnos perciben como positivo el rol de cada uno de ellos (puntaje 29.55); en la dimensión de necesidades, tuvieron un puntaje de 30.35, el cual se interpreta como la necesidad de mayor entrenamiento y enseñanza, y en cuanto al soporte social, lo consideran como un ambiente con más pros que en contra (puntaje 25.55).

Sin embargo, al considerar por separado los diferentes grados, sólo los residentes de tercer año consideraron que su ambiente educativo es más favorable que negativo. Por su parte, los de primer

Tabla 1: Características generales $(n=52)$.

\begin{tabular}{lc}
\hline Característica & $\mathrm{n}(\%)$ \\
\hline $\begin{array}{l}\text { Edad (media } \pm \mathrm{DE}) \\
\text { Sexo }\end{array}$ & $30.81 \pm 3.4$ \\
$\quad$ Hombres & $24(46.2)$ \\
$\quad$ Mujeres & $28(53.8)$ \\
Estado civil & \\
$\quad$ Soltero & $18(34.6)$ \\
Casado & $29(55.8)$ \\
$\quad$ Unión libre & $5(9.6)$ \\
Grado de residencia & \\
$\quad$ Primer año & $21(40.4)$ \\
Segundo año & $19(36.5)$ \\
$\quad$ Tercer año & $12(23.1)$ \\
\hline
\end{tabular}


Tabla 2: Ambiente educativo por año de residencia.

\begin{tabular}{lcccc} 
& Primer año & Segundo año & Tercer año & P* $^{*}$ \\
\hline Puntaje total & 80.49 & 77.21 & 102.5 & \\
Media \pm DE & $2.023 \pm 0.49$ & $1.9303 \pm 0.47$ & $2.5688 \pm 0.40$ & 0.0001 \\
\hline
\end{tabular}

*ANOVA de una vía.

y segundo año lo perciben como un ambiente educativo con muchos problemas, encontrando una diferencia significativa entre los tres grupos (Tabla 2).

Maltrato académico y físico. En el análisis global, sólo 15\% de los residentes aceptó haber recibido algún tipo de maltrato. Sin embargo, al separarse por año de residencia, se encontró un incremento en los médicos residentes de tercer año, quienes manifestaron haber recibido maltrato (25.6\%); cabe destacar que ninguno aceptó haber tenido guardias de castigo, pero sí fue muy frecuente el rubro de «haber recibido gritos». La fuente de estas agresiones provino principalmente de los médicos adscritos (Tabla 3).

\section{DISCUSIÓN}

La evaluación del ambiente educativo por parte de los residentes de medicina familiar del presente estudio, en general, es buena, aunque es menor a lo reportado por otros estudios, como el de Herrera y su equipo ${ }^{75}$ y el estudio de Llera y Durante. ${ }^{26}$ No obstante, también es semejante a otros como el de Concepción y colaboradores, quienes evaluaron el ambiente educativo de cinco hospitales en médicos internos de pregrado, obteniendo resultados diferentes según cada hospital de estudio (entre 63 y 90 de puntaje global), ${ }^{27}$ así como el de Hechenleitner y su equipo. ${ }^{28}$ Lo mismo se repite en los tres dominios, donde se muestra que hay disparidad en cuanto a los resultados. Esto explica la naturaleza de la escala en sí y lo que se intenta medir (ambiente educativo), que es influenciado por varios factores, en especial, el lugar y el tiempo, pero también el grado de estudios en los que se aplica, lo que justifica que se continúen haciendo análisis semejantes, ya que los resultados son dependientes de las características locales.
En cuanto al maltrato, se esperaba una frecuencia más alta en los diferentes estudios (la frecuencia es hasta de 80\%); ;1,22 sin embargo, en nuestro estudio apenas alcanzó 15\%; esto se puede justificar de dos formas: la primera explicación tiene que ver con la escala utilizada, pues, cuando se usan escalas más amplias, suele haber un porcentaje mayor de aceptación de maltrato; la otra es porque el alumno tiene introyectada la violencia (violencia simbólica), ${ }^{8}$ así como el temor de que aceptar dicho maltrato puede traer como consecuencia alguna sanción.

Finalmente, una mayoría aceptó por lo menos algún tipo de maltrato, en especial verbal, cuya fuente proviene por parte de los médicos adscritos.

\section{CONCLUSIONES}

En el presente estudio, los médicos residentes perciben a su ambiente educativo más positivo que negativo. El puntaje global obtenido fue de 85.38 con una necesidad de mejor capacitación. No obstante, al separar los resultados por año de residencia, los de primer y segundo año consideraron su ambiente con muchos problemas. En cuanto al maltrato, la frecuencia es baja, pues apenas registra un porcentaje que va desde 15\%

\begin{tabular}{lc}
\multicolumn{2}{c}{$\begin{array}{c}\text { Tabla 3: Percepción de maltrato } \\
\text { global y por año de residencia. }\end{array}$} \\
\hline \% de maltrato $(\mathrm{n}=52)$ \\
\hline Global & 14.2 \\
Primer año & 9.2 \\
Segundo año & 9.5 \\
Tercer año & 25.6 \\
\hline
\end{tabular}


global y se incrementa en el tercer año de residencia hasta $25.6 \%$.

Si bien los resultados no son tan malos como se esperaba, es necesario continuar con una política de mejora de sus ambientes de aprendizaje, así como de no tolerancia a la violencia contra los residentes.

\section{AGRADECIMIENTOS}

A todos los residentes voluntarios por su participación.

\section{BibliografíA}

1. Galli A, Brissón ME, Soler C, Lapresa S, Alves-de Lima A. Evaluación del ambiente educacional en residencias de cardiología. Rev Argent Cardiol. 2014; 82: 396-401. Disponible en: http://dx.doi.org/10.7775/rac.es.v82.15.3875

2. Castro-Rodríguez Y, Lara-Verástegui R. Percepción del ambiente educacional por estudiantes del pregrado en odontología. Rev ADM. 2017; 74 (3): 133-140. Disponible en: https://www.medigraphic.com/cgi-bin/new/resumen. cgi?IDARTICULO=72667

3. Vásquez N, Peralta J. Ambiente educativo clínico al final del internado en la escuela de medicina de la Universidad de Chile: resultados de la encuesta PHEEM. Rev Hosp Clin Univ Chile. 2013; 24: 193-201. Disponible en: https://www. researchgate.net

4. Mejía R, Diego A, Alemán M, Maliando MR, Lasala F. Percepción de maltrato durante la capacitación de médicos residentes. Medicina. 2005; 65: 295-301. Disponible en: https://www.medicinabuenosaires.com

5. Cumplido-Hernández G, Campos-Arcineaga MA, Chávez-López A. Significado de las relaciones laboralesinterpersonales en médicos residentes. Rev Med Inst Mex Seguro Soc. 2007; 45 (4): 361-369. Disponible en: https:// www.medigraphic.com/pdfs/imss/im-2007/im074h.pdf

6. Hernández-Pérez F. Grado de aceptación de la violencia simbólica de profesores y estudiantes del IMSS. Arch Med Urgen Mex. 2016; 8 (1-2): 41-47. Disponible en: https://imbiomed.com.mx/1/1/articulos. php? method=showDetail\&id_articulo $=107773 \& i d \_$ seccion=5639\&id_ejemplar=10483\&id_revista=359

7. Hernández-Pérez F, Castillo-Altamirano TA, MendozaMurillo K. Efecto de las guardias en habilidades cognitivas en estudiantes de especialidades médicas y médicos internos de pregrado. Rev CONAMED. 2017; 22 (1): 18-22. Disponible en: https://www.medigraphic.com/cgi-bin/new/ resumen.cgi?IDARTICULO=79344

8. Bourdieu P, Passeron JC. Fundamentos de una teoría de la violencia simbólica. En: La reproducción. Elementos para una teoría del sistema de enseñanza. México: Fontamara; 2005. pp. 16-108.

9. Secretaría de Salud, Estados Unidos Mexicanos. (04 de 01 de 2013). Norma Oficial Mexicana NOM-001-SSA3-2012. Distrito Federal, México.

10. Sepúlveda-Vildósola AC, Mota-Nova AR, Fajardo-Dolci GE, Reyes-Lagunes I. Acoso laboral durante la formación como especialista en un hospital de pediatría en México: un fenómeno poco percibido. Rev Med Inst Mex Seguro Soc. 2017; 55 Supl 1: S92-S101. Disponible en: https://www.medigraphic.com/cgi-bin/new/resumen. cgi?IDARTICULO=72055

11. Derive S, Casas-Martínez ML, Obrador-Vera GT, Villa AR, Contreras D. Percepción de maltrato durante la residencia médica en México: medición y análisis bioético. Inv Ed Med. 2017; 1-8. Disponible en: http://dx.doi.org/10.1016/j. riem.2017.04.004

12. Carrillo-Esper R, Gómez-Hernández K. Bullyng durante el pre y postgrado de la formación médica. Rev Invest Med Sur Mex. 2014; 21 (4): 172-176. Disponible en: https://www. medigraphic.com/pdfs/medsur/ms-2014/ms144d.pdf

13. Peña-Saint F, López-Molina J. Acoso docente: maltrato psicológico de profesores hacia alumnos contextos universitarios tóxicos. Revista Ra Ximha. 2016; 12: 33-47. Disponible en: https://docplayer.es/9265108-Acosodocente-maltrato-psicologico-de-profesores-haciaalumnos-en-contextos-universitarios-toxicos.html

14. Hamui-Sutton A, Flores-Hernández F, Gutiérrez-Barreto S, Castro-Ramírez S, Lavalle-Montalvo C, Vilar-Puig P. Correlaciones entre las dimensiones de los ambientes clínicos de aprendizaje desde la percepción de los médicos residentes. Gaceta Médica de México. 2014; 150: 144-153. Disponible en: https://www.anmm.org.mx/GMM/2014/n2/ GMM_150_2014_2_144-153.pdf

15. Herrera C, Olivos T, Román JA, Larraín A, Pizarro M, Solis $\mathrm{N}$ et al. Evaluación del ambiente educacional en programas de especialización médica. Rev Med Chile. 2012; 140: 1554-1561. Disponible en: https://scielo.conicyt.cl/ scielo.php?pid=S0034-98872012001200006\&script $=$ sci_ abstract\&tlng=pt

16. Conzález-Cabanach R, Valle-Arias A, Núñez-Pérez JC, González-Pienda JA. Una aproximación teórica al concepto de metas académicas y su relación con la motivación escolar. Psicothema. 1996; 8 (1): 45-61. Disponible en: http:// www.redalyc.org/articulo.oa?id=72780104

17. Díaz-Vélis G, Mora S, Escanero JF. Percepción del ambiente educacional en dos escuelas de medicina con currículo tradicional. Estudio longitudinal. Rev Med Chile. 2016; 144: 1479-1485. Disponible en: https://scielo.conicyt.cl/pdf/rmc/ v144n11/art15.pdf

18. Ríos-González CM. Percepción del ambiente educativo en estudiantes de medicina de Latinoamérica, 2015. CIMEL. 2016; 21 (2): 38-41. Disponible en: https://www.cimel. felsocem.net/index.php/CIMEL/article/view/640 
19. Chavarria-Islas R, Rivera-Ibarra D. Entorno laboral y aptitudes clínicas en residentes de urgencias médicoquirúrgicas. Rev Med IMSS. 2004; 42 (5):371-378. Disponible en: https://www.medigraphic.com/cgi-bin/new/resumen. cgi?IDARTICULO=10424

20. Cárdenas-Ayón E, Cabrera-Pivaral CE, Viniegra-Velázquez L, González-Rey SHF, Luce-González EG, Ortiz-López MA. Satisfacción de los residentes de medicina familiar con el ambiente académico laboral. Rev Med Inst Mex Seguro Soc. 2010; 48 (2): 227-231. Disponible en: https://pdfs. semanticscholar.org/ble4/547884dacd505d754b8518a11 613a7c34730.pdf

21. Herrera-Silva JC, Treviño-Moore A, Acosta-Corona CO. Características de la violencia en residentes de dos instituciones formadoras de pediatras en el estado de Sonora. Bol Med Hosp Infant Mex. 2006; 63: 18-30. Disponible en: http://www.scielo.org.mx/pdf/bmim/v63nt/ v63nla4.pdf

22. Ortiz-León S, Jaimes-Medrano AL, Tafoya-Ramos SA, Mujica-Amaya ML, Olmedo-Canchola VC, CarrascoRojas JA. Experiencias de maltrato y hostigamiento en médicos residentes. Cir Cir. 2014; 82: 290-301. Disponible en: https://www.medigraphic.com/pdfs/circir/cc-2014/ ccl43h.pdf

23. Consejo C, Viesca-Treviño C. Ética y relaciones de poder en la formación de médicos residentes e internos: algunas reflexiones a la luz de Foucault y Bourdieu. Bol Mex His Fil Med. 2008; 11 (1): 16-20. Disponible en: https://www.medigraphic.com/cgi-bin/new/resumen. cgi?IDARTICULO=15332

24. Miní E, Medina J, Peralta V, Rojas L, Butron J, Gutiérrez EL. Programa de residentado médico: percepciones de los médicos residentes en hospitales de lima y callao. Rev Peru Med Exp Salud Pública. 2015; 32 (2): 303-310. Disponible en: http://www.scielo.org.pe/pdf/rins/v32n2/ a15v32n2.pdf

25. Ríos-Nava B, Romero-Mariscal SL, Olivo-Estrada JR. La percepción del maltrato de las y los estudiantes nayaritas en las carreras universitarias de medicina enfermería. Un primer acercamiento. Waxapa. 2013; 5 (9):20-30. Disponible en: https://www.medigraphic.com/cgi-bin/new/resumen. cgi?IDARTICULO=50930

26. Llera J, Durante E. Correlación entre el clima educacional y el síndrome de desgaste profesional en los programas de residencia de un hospital universitario. Rev Hospital Italiano de Buenos Aires. 2013; 1-10. Disponible en: http:// dx.doi.org/10.5546/aap.2014

27. Concepción-Gómez RA, Asprilla-González JA. Aplicación del cuestionario PHEEM a médicos internos en 5 hospitales de Panamá. Inv Ed Med. 2017. Disponible en: http://dx.doi. org/10.1016/j.riem.2017.04.007

28. Hechenleitner M, Flores G, Bittner V, Alarcón M, Meyer A, Lermanda C. Percepción del clima educacional en los internados de Medicina de la Universidad Católica de la Santísima Concepción. Rev Educ Cienc Salud. 2015; 12 (2): 150-156. Disponible en: www.udec.cl 